\title{
Innovationskraft trotz Vetospieler: Bildungspolitische Reformen im deutschen Föderalismus
}

Teile der deutschen Bildungspolitik, insbesondere in der Hochschul- und Berufsbildung, gelten als Paradebeispiele einer zähen Politikverflechtung zwischen Bund und Ländern. Wegen ihrer hohen Zahl an Vetospielern steht diese typisch deutsche Aushandlungsstruktur unter dem Verdacht, nötige Reformen zu blockieren und den politischen Wandel auf einem zentralen gesellschaftlichen Feld zu verhindern. Ein Blick auf bislang kaum analysierte Reformprojekte zeigt jedoch: Die föderale Konfliktarena verkompliziert zwar das Reformieren, aber sie vermag es auch, die Reformen innovativer zu machen.

CHRISTIAN FÖRSTER, JOHANNES KLENK

\section{Einleitung}

Bildungspolitik ist während der vergangenen zehn Jahre, ob ihrer gesellschaftlichen Relevanz richtigerweise, zum Modethema der politischen und sozialwissenschaftlichen Diskussion geworden. Regelmäßig veröffentlichte internationale Bildungsvergleiche wie etwa die PISA-Studien oder „Education at a Glance“ verursachen erheblichen Aufruhr in der Debatte und belegen für Deutschland hohen Reformbedarf. Auch abseits dieser rein diskursiven Ebene ändert sich einiges, sind doch die verschiedenen Bildungsbereiche Gegenstand erheblicher Reformbemühungen. Zahlreiche Studien thematisieren aktuelle politische Gestaltungsanstrengungen, die im Zusammenhang mit europäischen und internationalen Einflüssen gesehen werden (vgl. exemplarisch Busemeyer 2009; Powell et al. 2012; Witte 2006 sowie als Überblick Busemeyer/Trampusch 2011). Bei der Beurteilung der Reformanstrengungen darf nicht aus den Augen verloren werden, dass wichtige Teile der Bildungspolitik nach wie vor als typischer Fall von Politikverflechtung im föderalen Entscheidungssystem gelten. An diesem Umstand scheiden sich die Geister: Einerseits wird kaum bestritten, dass der kooperative Verbundföderalismus in Deutschland zu zähen Aushandlungsprozessen neigt (klassisch: Scharpf 1985 sowie Tsebelis 2002). Andererseits sorgen in manchen Fällen Mechanismen der Konsensfindung und das konstruktive Verhalten der beteiligten Akteure dafür, dass die komplizierten und langwierigen Politikprozesse im Verbundföderalismus dennoch Ergebnisse produzieren (etwa: Bandelow 2005; Benz 2003). Es ist unklar, welche dieser Varianten auf aktuelle, im Föderalismus ausgehandelte Umgestaltungen des Bildungsbereichs eher zutrifft.

Vor dem Hintergrund dieser gewissen Ratlosigkeit über die bildungspolitische Reformbilanz in Deutschland widmet sich der vorliegende Artikel zwei bislang wenig analysierten Reformprozessen. In vergleichender Perspektive werden die Entschließung der Exzellenzinitiative für Hochschulen und die deutsche Umsetzung des Europäischen Qualifikationsrahmens (EQR) gegenübergestellt. Im Mittelpunkt steht dabei die Frage, ob durch die komplizierten Interaktionsprozesse zwischen Akteuren auf verschiedenen Ebenen des Föderalismus der hervorgerufene Politikwandel beschränkt wurde.

Der folgende Abschnitt 2 wird zunächst auf genereller Ebene Politikverflechtung und die Rolle von Vetospielern im Föderalismus beleuchten. Anschließend widmen sich zwei kurze Fallstudien der Exzellenzinitiative (3) sowie der EQR-Umsetzung (4), bevor wir im letzten Teil des Artikels ein Fazit (5) ziehen. Die empirische Grundlage beider Fallstudien stellen Experteninterviews mit Vertretern der direkt an den Reformprozessen beteiligten Akteure dar (zur Methode siehe Meuser/Nagel 2009). Für den Prozess zur Exzellenzinitiative wurden acht Personen aus den für Hochschulen verantwortlichen Ministerien des Bundes sowie ausgewählter Länder und ein Vertreter einer zentralen In- 
teressengruppe in der Hochschulpolitik befragt. Die Erkenntnisse zur Entwicklung des Deutschen Qualifikationsrahmens stammen aus acht Expertengesprächen mit Personen aus Sozialpartnerverbänden, Bundesministerium für Bildung und Forschung (BMBF) und Kultusministerkonferenz (KMK). Die Gespräche nahmen zwischen 20 und 90 Minuten ein, wurden im Zeitraum von November 2009 bis November 2010 geführt, danach vollständig transkribiert und EDV-gestützt analysiert.

\section{Wie innovationsfähig ist Föderalismus?}

Die Frage, wie sich der deutsche Föderalismus auf die Innovationsfähigkeit politischer Reformen auswirkt, ist umstritten. In einer lebendig geführten Forschungsdebatte, die wir hier nur ausschnittsweise wiedergeben, stehen sich verschiedene Argumente gegenüber.

Einerseits hat die Ausgestaltung des Bundesstaats nach Meinung vieler Beobachter „zur Stabilisierung der Verhältnisse beigetragen“ (Hrbek 2001, S. 55). Die im deutschen Föderalismus bestehenden Zwänge zur Verhandlung sorgen demnach dafür, dass verschiedene Positionen in den Politikprozess integriert werden. Nicht zuletzt verbindet sich hiermit die Erwartung, die sogenannte „Externalitätsproblematik“, eine Konkurrenz des „unmittelbare[n] Eigeninteresse[s] der dezentralen Einheiten mit dem Gesamtinteresse" (Scharpf et al. 1976, S. 46), zu entschärfen.

Allerdings bietet die Ausgestaltung des deutschen Bundesstaats als kooperativer, verflochtener Verbundföderalismus daneben Anlass zu kritischen Anmerkungen. So erkennt Scharpf einen problematischen Dualismus aus sachpolitischer Handlungsnotwendigkeit und hohen Hürden zur Konsensherstellung (Scharpf et al. 1976; siehe als aktualisierte Fassung auch Scharpf 2009). Gelingt es nicht, diesen Widerspruch durch die Anwendung „effektiver Konsensbildungsstrategien“ (Scharpf et al. 1976, S. 54) aufzulösen, entwickelt sich die Politikverflechtung zu einer „Politikverflechtungsfalle“ (Scharpf 1985), aus der sich die beteiligten Akteure aufgrund des Einstimmigkeitsprinzips nur schwerlich befreien können. Dieser These stimmt auch der sogenannte Vetospieler-Ansatz (Tsebelis 2002) zu. Dort wird durch theoretische Modellierung gezeigt, dass insbesondere Föderalismen mit einem stark ausgeprägten Bikameralismus (wie Deutschland) durch eine hohe Zahl an verhinderungsmächtigen Akteuren gekennzeichnet sind. Föderalstaaten weisen daher ein hohes Maß an „policy stability“ (Tsebelis 2002, S. 136) auf: Innovative Reformen sind hier vergleichsweise schwer zu erreichen.

\subsection{Konstruktive Vetospieler und freiwillige Verflechtung}

In den vergangenen rund zehn Jahren verlagerte sich der Fokus der Debatte auf Faktoren, die trotz der problematischen Ausgangslage Konsensbildung ermöglichen. Hierbei verwies beispielsweise Benz (2003) auf „konstruktive Vetospieler": Demnach nehmen föderale Akteure ihre Macht strategisch wahr und wägen den Einsatz eines Vetos gegen die Kosten einer Reformblockade, wie etwa einer öffentlichen Schuldzuweisung auf den „Blockierer“, ab (Benz 2003, S. 212). Hierzu passend zeigte Bandelow (2005), dass der Entscheidungsverbund mächtiger Akteure ein Umdenken bei der politischen Problemlösung nicht blockieren muss, sondern dieses sogar stimulieren kann. Gestützt wird diese Argumentation nicht zuletzt durch empirische Beispiele für freiwillige Verflechtung, in deren Rahmen sich Akteure für Verhandlungen entscheiden, auch wenn diese verfassungsmäßig gar nicht zwingend sind (Benz 2009, S. 13ff.).

\subsection{Sonderfall Bildungsföderalismus}

Die Bildungspolitik nimmt in der Föderalismusdebatte einen besonderen Platz ein. Das Politikfeld gilt aus Sicht der Landesregierungen als wichtige Bastion der Zuständigkeit, nach Meinung mancher Autoren gar als „sakrosankt“ (Kreft 2006, S. 15). Von Kritikern als Relikt aus Zeiten der Kleinstaaterei geschmäht, wird der Bildungsföderalismus als verantwortlich für verschiedenste Probleme im deutschen Bildungssystem identifiziert. Mit der Institution Kultusministerkonferenz existiert eine ausgeprägte Koordinierungsinstanz für die verschiedenen Länderpolitiken, insgesamt herrscht ein hohes Maß an Politikverflechtung. Die Steuerungsreformen der 1960er und 1970er Jahre haben deutliche Spuren in Form von Zwangsverhandlungsstrukturen hinterlassen, die auch die Föderalismusreform des Jahres 2006 nur teilweise abbauen konnte (Sandberger 2008; Münch 2009). Im Steuerungsarrangement der Berufsbildung ist der an sich schon nicht einfache Bildungsföderalismus zudem noch stark mit korporatistischen Elementen durchsetzt. Auf allen Ebenen der deutschen Berufsbildung existieren paritätisch mit beiden Sozialpartnerseiten besetzte Steuerungsinstitutionen. Die in staatlicher Verantwortung organisierte Berufsschule hat steuerungsseitig allenfalls den Status eines Juniorpartners (Baethge 2008, S. 546).

Mit Blick auf aktuelle Reformen des Bildungsbereichs drängen sich aus dieser Perspektive Fragen auf: Welche der erörterten Auswirkungen des Verbundföderalismus macht sich bemerkbar? Haben bildungspolitische Reformen in Deutschland unter Blockadesituationen gelitten? Wäre, rein hypothetisch, in einem unilateral geprägten Entscheidungssystem mehr Innovation möglich gewesen? Empirische Prozessanalysen hierzu fehlen weitgehend. Zwar stellen aktuelle Einschätzungen zum deutschen Bildungsföderalismus wertvolle Analysen der institutionellen Aus- und Umgestaltung des Entscheidungssystems dar, diese überprüfen 
die Innovationskapazität des Systems allerdings nicht durch eine detaillierte Betrachtung von tatsächlichen Politikergebnissen (siehe etwa Griessen/Braun 2010, S. 726ff., 735ff.; ferner Sandberger 2008; Münch 2009).

Die folgende Betrachtung soll helfen, diese Lücke zu schließen: An den Beispielen der Exzellenzinitiative und der deutschen Umsetzung des Europäischen Qualifikationsrahmens zeichnen wir nach, welche Zusammenhänge zwischen der Interaktion von Vetospielern auf Bundes- und Länder-Ebene und zwei aktuellen Politikergebnissen im Bildungsbereich bestehen. Mit dieser Auswahl von Untersuchungsfällen wird ein kontrollierter Vergleich hinsichtlich bestimmter Schlüsselfaktoren in der föderalen Interaktion realisiert - die folgenden Fallstudien geben hierzu nähere Auskunft.

\section{Die Exzellenzinitiative: Einigung dank monetärer Anreize und gemeinsamer Reformvorstellungen}

Im Rahmen der Exzellenzinitiative werden seit dem Jahr 2006 Konzepte zur Forschung an Hochschulen aus Bundesund Landesmitteln (Aufteilung: 75 \% Bund, 25 \% Sitzland) gefördert. Die drei Förderlinien umfassen Graduiertenschulen zur Doktorandenausbildung, thematische Netzwerke unter Beteiligung außeruniversitärer Forschungseinrichtungen (sog. Exzellenzcluster) und Zukunftskonzepte zur Entwicklung der gesamten Hochschule (häufig als „Eliteuniversitäten“ bezeichnet). Grundlage für Beschließung und Durchführung der Exzellenzinitiative ist die gemeinsame Forschungs- und Wissenschaftsförderung von Bund und Ländern nach Art. 91b Grundgesetz (GG) (alte und neue Fassung). Der Entscheidungsprozess zur Exzellenzinitiative stand also mitten im ,institutionellen und prozessualen Rahmen des kooperativen Föderalismus“ (Münch 2009, S. 225)

Ins Gespräch gebracht wurde die Idee zur besonderen Förderung von Spitzenhochschulen zu Beginn des Jahres 2004 aus dem Umfeld des damaligen SPD-Bundeskanzlers Gerhard Schröder (vgl. SPD 2004, S. 6). Der Impuls wurde alsbald vonseiten des BMBF unter Führung der damaligen Ministerin Edelgard Bulmahn (SPD) aufgegriffen und in die Bund-Länder-Verhandlungen im Rahmen der Bund-Länder-Kommission für Bildungsplanung und Forschungsförderung überstellt (Pasternack 2008, S. 15f.).

\subsection{Konflikt trotz grundsätzlich geteilter Reformvorstellungen}

Das grundsätzliche Ziel hinter dem Vorschlag des Bundes, Spitzenforschung nicht nur zu fördern, sondern diese auch stärker als bisher öffentlich sichtbar zu machen, wurde von den Ländern begrüßt. Verschiedene Interviewpartner beschreiben dieses Konzept als zu ihrer Vorstellung zeitgemäßer Hochschulpolitik passend.

„[D]ie Idee ist [ ...], dass wir einen erkennbaren und auch nicht zu leugnenden internationalen Wettbewerb haben. Das, was wir als Globalisierung in anderen Bereichen des Lebens kennen, haben wir natürlich in der Wissenschaftspolitik auch. [...] Insofern dann sich die Frage zu stellen, wie schaffen wir es selbst, Konkurrenzfähigkeit auch zu sichern, wie schaffen wir es, [...] Sichtbarkeit [...] zu erhöhen, dass wir auf der einen Seite zwar eins der größten, potentesten Länder sind, aber vielleicht trotzdem durch unsere föderale Struktur nicht unbedingt jede einzelne größere Universität erkennbar ist, weil sie unter einem Landeslabel läuft und nicht sozusagen unsere Deutschlandflagge drüber hängt." (Policy-Experte Exzellenzinitiative).

$\mathrm{Zu}$ einer solchen Einschätzung gelangten, dies zeigen unsere Interviews, durchaus auch Vertreter jener Länder, die in den tatsächlichen Vergaberunden der Folgejahre leer ausgehen sollten. Der Politikprozess war also von Anfang an durch eine recht homogene Präferenzlage über die Ziele der Forschungsförderung bei Bund und Ländern geprägt. Neben dieser Gleichartigkeit von abstrakten Konzepten über "gute“Wissenschaftspolitik unter den Verhandlungsteilnehmern war eine weitere günstige Ausgangsbedingung für den Entscheidungsprozess die früh getroffene Zusage des Bundes, einen Großteil der Kosten zu übernehmen. Diese Aussicht stellte zweifelsohne einen hohen Anreiz zur Einigung dar - einer der Gesprächspartner spricht hier von einer „Beutegemeinschaft" der Länder.

Dennoch waren die Verhandlungen konfliktreich (vgl. Förster 2011, S. 121ff., S. 212). Zu Beginn umfasste der Vorschlag des Bundes lediglich die Förderung ganzer Hochschulen (später Förderlinie Zukunftskonzepte). Die Länder standen diesem Zuschnitt des Projekts jedoch kritisch gegenüber. Der Konflikt übertönte schnell jede parteipolitische Konnotation der Debatte und ließ stattdessen deutlich eine Bruchlinie zwischen Bund und Ländern hervortreten:

„Die Länder sind dann [...] sehr geschlossen gelaufen an der Konfliktlinie des Bundes entlang, sodass es eigentlich eine Verhandlung zwischen Bund und Ländern war." (Policy-Experte Exzellenzinitiative)

Unsere Interviews weisen auf zwei Gründe für die ablehnende Haltung der Länder hin. Erstens befürchteten die Landesregierungen aufgrund vorhandener Erfahrung in der Forschungsförderung eine regionale Konzentration der Fördermittel im Süden. Zweitens bestanden in den Fachabteilungen einiger Landesministerien Vorbehalte gegen die Idee, Elite-Universitäten überhaupt durch eine bewusste Entscheidung identifizieren zu können. Vielmehr sollten sich diese über einen natürlichen Prozess herausbilden.

Nachdem eine mit den Verhandlungen betraute Arbeitsgruppe von Staatssekretären diese Vorbehalte nicht ausräumen konnte, schien eine Einigung trotz der auf abstrakter Ebene homogenen Präferenzlage und der monetären Anreize für potenzielle Gewinnerländer der Initiative gefähr- 
det. Als alternative Konsensfindungsstrategie wurde daher ein lediglich dreiköpfiges Aushandlungsgremium mit je einem Vertreter von Bund, A- und B-Ländern eingesetzt. Hier wurde die schlussendlich zu projektierende Drei-Säulen-Struktur entwickelt (vgl. Bundesregierung et al. 2005). Durch diese wurde zum einen die Befürchtung einer möglichen regionalen Förderkonzentration abgeschwächt. Zweitens ließ die Vereinbarung, geförderte Zukunftskonzepte bei künftigen Vergaberunden nach outputorientierten Kriterien erneut zu beurteilen, die Grundsatzkritik an einer bewussten Bestimmung einzelner Spitzenhochschulen in den Hintergrund treten. Die hohe Konsensfähigkeit dieses Konzepts zeigten auch die heftigen Gegenreaktionen der übrigen Länder, als im Juni 2005 der damalige hessische Ministerpräsident Roland Koch (CDU) ankündigte, seine Zustimmung zu verweigern.

\subsection{Inhaltliche Ausweitung durch konstruktive Vetospieler}

Während des Politikprozesses schränkte die bestehende Zwangsverhandlung, im Vergleich zu einer freilich rein hypothetischen unilateralen Lösung, den Handlungsspielraum der beteiligten Akteure also ein. Der Prozess wurde so verkompliziert und gebremst - ein Vorgang, der den grundsätzlichen Hypothesen der oben skizzierten theoretischen Arbeiten zur Politikverflechtung und zu Vetospielern entspricht (Scharpf et al. 1976; Scharpf 1985; Tsebelis 2002). Allerdings schlägt sich diese „Abbremsung“ nicht in einer Herabsetzung des Innovationsgrades beim Politikergebnis nieder: Der zunächst einseitig vom Bund entwickelte Vorschlag fand schließlich Eingang in die finale Form der Exzellenzinitiative. Im Aushandlungsprozess wurde er durch weitere Elemente der Umgestaltung (Graduiertenschulen und Exzellenzcluster) ergänzt. Die Intensität der Reform, also das Ausmaß des verursachten Wandels, wurde so durch die Bund-Länder-Verhandlungen nicht verringert, sondern gesteigert.

In der öffentlich geführten Debatte mag die Exzellenzinitiative als zur Anwendung kommende Policy umstritten sein - v. a. vonseiten der Angehörigen des Hochschulsystems (z. B. Hartmann 2006; Münch 2007). Misst man das Politikergebnis jedoch an seiner problematischen Entstehungsgeschichte, kann von einer gewissen Zufriedenheit gesprochen werden. Kaum verwunderlich ist dabei, dass die Vertreter von Gewinner-Ländern der Initiative anführen, die Reform habe „eine Menge in Gang gesetzt [...]. [I]n den Hochschulen, sowohl in den Exzellenzhochschulen als auch in den anderen, sind Kräfte aktiviert worden, die vorher schlummerten." (Policy-Experte Exzellenzinitiative).

Aber auch Angehörige jener Länder, die bei der tatsächlichen Mittelvergabe schlecht abschnitten, beurteilen die jetzige Form der Initiative positiver als den ursprünglich von BMBF-Seite initiierten Reformvorschlag - trotz vorgetragener genereller Ergebniskritik und Nachbesserungen wie etwa der Landesexzellenzinitiative in Sachsen. Auch unter diesem Blickwinkel hat die Initiative durch den ihr vorausgegangenen komplizierten Aushandlungsprozess zwischen Bund und Ländern also durchaus gewonnen.

Insgesamt zeigt die Exzellenzinitiative damit die Innovationsfähigkeit der zwischen Bund und Ländern verflochtenen deutschen Bildungspolitik. Was in der geschilderten Entstehungsgeschichte der Initiative allerdings ebenso auffällt, ist die Tatsache, dass bereits bei den Startbedingungen der Verhandlungen einige Weichen günstig gestellt waren. Wie oben gezeigt, teilten die entscheidenden Akteure auf Länderebene prinzipiell das Handlungsziel des Bundes, die Sichtbarkeit von Spitzenforschung zu erhöhen. Ein Verhalten als „konstruktive Vetospieler“ (Benz 2003) war damit prädisponiert. Mit der Finanzierungszusage durch den Bund bestanden zudem hohe monetäre Einigungsanreize.

Vor diesem Hintergrund erscheint es lohnenswert, die Betrachtung der Exzellenzinitiative durch einen bildungspolitischen Fall zu ergänzen, in dem die Ausgangsbedingungen (sowohl hinsichtlich normativer Einstellungen als auch hinsichtlich monetärer Anreize) ungünstiger sind. Auf die deutsche EQR-Umsetzung trifft dies zu. Die folgende Fallstudie kann daher zeigen, ob es sich bei den begünstigenden Faktoren, die im Zusammenhang mit der Exzellenzinitiative zum Tragen kamen, wirklich um notwendige Bedingungen einer bildungspolitischen Reform unter der Beteiligung föderaler Vetospieler handelt - oder ob sich die föderale Interaktion nicht auch unter ungünstigeren Bedingungen positiv auf Politikergebnisse auswirkt.

\section{Die deutsche EQR-Umsetzung: Einigung dank komplexer Governance}

Mit dem Deutschen Qualifikationsrahmen wird derzeit ein neues Steuerungsinstrument in die deutsche Bildungspolitik eingeführt, dessen Intention die einheitliche Beschreibung von Bildungsergebnissen in einer achtstufigen Skala ist. Der Prozess geht auf eine europäische Initiative zurück und wurde im Jahr 2008 mit der Verabschiedung eines gemeinsamen Europäischen Qualifikationsrahmens (EQR) formal gestartet (Europäisches Parlament und Rat 2008). Ausgangspunkt ist der Bereich der Berufsbildung, letztlich werden aber alle Bildungsbereiche adressiert. In der föderalen Aufgabenverteilung in Deutschland sind damit wie bei der Exzellenzinitiative Kompetenzbereiche sowohl des Bundes als auch der Länder berührt. Wenn, wie dort, eine gemeinsame Lösung erreicht werden soll, dann erzwingen geteilte Kompetenzen Verhandlungen (vgl. Benz 2009, S. 13). BMBF und KMK als zuständige Akteure beider Ebenen haben sich in diesem Fall darauf verständigt, die Verantwortung für die Entwicklung und Einführung eines deutschen Qualifikationsrahmens gemeinsam wahrzunehmen. Dazu wurde eigens eine Bund-Länder-Koordi- 
nierungsgruppe eingerichtet, deren Zusammensetzung aus unterschiedlichen Akteuren beider Ebenen deutlich die komplexe, durch die Verfassung erzwungene Politikverflechtung in Fragen der bildungspolitischen Steuerung zeigt.

Der EQR enthält keine Angaben zur konkreten Gestalt nationaler Qualifikationsrahmen; dort ist vielmehr festgehalten, dass Besonderheiten der nationalen Bildungssysteme so weit als möglich erhalten bleiben sollen. Aus den erheblich divergierenden Gestaltungsvorstellungen der beteiligten Ministerien des Bundes wie der Länder resultierte auf der inhaltlichen Ebene eine faktische Innovationsblockade: BMBF wie KMK schätzten eine Einigung untereinander als potenziell schwierig und aufwendig ein und verzichteten daher auf einen Abstimmungsprozess. Diese Situation verweist unseres Erachtens auf das in der spezifischen institutionellen Konstellation der Bundesrepublik zementierte Konfliktverhältnis der unterschiedlichen Bildungsbereiche: Die langjährige Trennung zwischen berufsorientiert-praktischer Ausbildung und akademisch-theoretischer (Hoch-)Schulbildung ist eben auch Ausdruck sozialer Selektivität. Die Nicht-Einigung belegt dabei eine mangelnde staatliche Gestaltungsfähigkeit, die für den Verlauf des weiteren Prozesses folgenreich war (vgl. Klenk 2011, S. 236).

Im Vergleich mit der oben behandelten Exzellenzinitiative erscheint diese Blockadesituation gleichwohl nachvollziehbar: Für die Hochschulreform konnten wir zweierlei begünstigende Bedingungen für den verflochtenen Aushandlungsprozess identifizieren: zum einen eine von Akteuren des Bundes und der Länderregierungen geteilte inhaltliche Hauptidee und zum anderen massive monetäre Anreize, die durch den Protagonisten der Reform (Bundesregierung) den übrigen Verhandlungspartnern (Landesregierungen) gesetzt wurden. Bei der EQR-Rezeption fehlten sowohl eine geteilte grobe Idee zur Lösung als auch die monetären Vorteile der Zustimmung.

\subsection{Konsenserzeugung durch Level-Shifting}

Um dem gemeinsamen Ziel eines bildungsbereichsübergreifenden Deutschen Qualifikationsrahmens (DQR) dennoch näher zu kommen, wurde die inhaltliche Entwicklungsarbeit in ein zweites Gremium, den sogenannten Arbeitskreis Deutscher Qualifikationsrahmen (AK DQR), verschoben. Dieses extra einberufene Gremium, in dem sich Bund- und Ländervertreter ebenfalls den Vorsitz teilen, integrierte eine Reihe nichtstaatlicher Akteure in die Entwicklungsarbeit. Hierin wird eine Strategie des „Level-Shifting“ sichtbar: Die Verlagerung in „ein Gremium mit speziellen Zuständigkeiten“ (Benz 2003, S. 221) lässt es zu, das Koordinationsproblem in einem veränderten Kontext neu zu definieren. Vertreter von KMK und BMBF sahen ihre Rolle fortan vorrangig in der Gewährleistung eines offenen und konstruktiven Diskussionsprozesses zwischen den übrigen am Prozess beteiligten Akteuren, wie beispielhaft an der folgenden Aussage deutlich wird:
„Ich sehe das schon als eine Moderatorenrolle, [...] wobei natürlich die Interessen der Länder von mir da eingebracht werden sozusagen. Aber moderierend in dem Sinne, dass mein Auftrag ja eher auch konkret ist, einen möglichst konsensualen Vorschlag vorzulegen." (Policy-Experte DQR).

Neben den beiden Vorsitzenden setzte sich der Arbeitskreis zu etwa einem Drittel aus Vertretern verschiedener Fachressorts auf Bundesebene zusammen, die sich im Verlauf der Entwicklungen wesentlich durch ihre Zurückhaltung auszeichneten. Zusammengenommen als stärkste Gruppe innerhalb des Gremiums traten die Vertreter der Berufsbildung in Erscheinung. Obwohl hier mit Gewerkschaften und Arbeitgeberverbänden zwei üblicherweise antagonistisch agierende Akteursgruppen vertreten waren, die auch in der Gestaltung der Berufsbildung divergierende Positionen vertreten (vgl. als umfassende Aufarbeitung Busemeyer 2009) und zudem in den beiden Lagern durchaus heterogene Interessenlagen zu verschiedenen Einzelthemen vereinen, erscheint es hier zweckmäßig, die Vertreter der Berufsbildung in Abgrenzung zu den anderen Bildungsbereichen in der Auseinandersetzung um einen Deutschen Qualifikationsrahmen als einheitlichen Akteur zu betrachten. Dies ist zulässig, weil die Berufsbildungsakteure sich in verschiedenen dem $A K D Q R$ vorgelagerten Gremien inhaltlich abgestimmt und ihr Handeln koordiniert haben. Strittige Punkte wurden bewusst ausgeklammert, womit „Strategiefähigkeit“ im Sinne Scharpfs (2000, S. 107ff.) erreicht werden konnte - offenkundig bot der Entwicklungsprozess genug Anreiz für ein koordiniertes Vorgehen.

Mit dem System der dualen Berufsausbildung und den Institutionen der tripartistischen Steuerung im Hintergrund verfügen die Vertreter der Berufsbildung über eine erhebliche Machtbasis. Für eine erfolgreiche Implementation des DQR war von vornherein ihre Zustimmung unabdingbar, sie sind daher grundsätzlich als (externer) Vetospieler zu betrachten. Mit der Einbeziehung in den Entscheidungsprozess wandelte sich dieser Charakter und man könnte sagen, dass hier eine Internalisierung wesentlicher externer Vetospieler stattfand. Dies ist bedeutsam, weil sich damit die Wahrscheinlichkeit destruktiven Vetospiels drastisch reduziert (vgl. Benz 2003, S. 213f.).

Mit Level-Shifting und Internalisierung der Berufsbildungsvertreter wurde also eine zweifache Änderung der komplizierten und zunächst zur Blockade führenden Rahmenbedingungen erreicht. Bemerkenswerterweise war die so entstandene Governance-Struktur nicht weniger verflochten als die Ausgangslage des Prozesses, jedoch handelte es sich nun um eine im Wesentlichen freiwillige Form der Verflechtung.

\subsection{Berufsbildung als Innovationsmotor}

Die vormalige Blockadesituation konnte so umgangen werden und hat sich zu einer durchaus innovationsfähigen Entwicklungsatmosphäre gewandelt, wie in den folgenden Monaten deutlich wurde: 
Während sich die staatlichen Akteure wie beschrieben auf eine Moderatorenrolle beschränkten, übernahmen die Vertreter der Berufsbildung zügig eine Führungsrolle in der Entwicklung, indem sie allen ursprünglichen Differenzen zum Trotz bereits vor der Einsetzung des Arbeitskreises einen konsolidierten Vorschlag vorlegten: [Wir] „haben auch schon einen ersten DQR-Vorschlag, parallel zu dem eingesetzten Bund-Länder-DQR-Arbeitskreis, entwickelt. Da haben wir dann schon unsere eigenen Beschreibungen entwickelt, der acht Stufen, auf die wir uns dann geeinigt haben, und haben auch Definitionen und Zielrichtung und so weiter definiert, [...um] die berufliche Bildung als Einheit im Konsenssinne auch zu positionieren, für den Zeitpunkt, als dann der DQR-Arbeitskreis seine Arbeit aufnahm“ (Policy-Experte DQR).

Sie verfolgten damit das klare Ziel, über den Deutschen Qualifikationsrahmen die empfundene Geringschätzung der Bildungsleistungen aus dem beruflichen Spektrum zu korrigieren. Umfangreiche Erfahrung aus der gemeinsamen Steuerung der dualen Berufsbildung, eine verlässliche Koalition der beteiligten Akteure und eine im Vergleich zu anderen non-gouvernementalen Akteuren großzügige Ausstattung mit Ressourcen garantierten eine hohe Handlungsfähigkeit. Die Repräsentation der Arbeitswelt als wichtigster Anwendungsbereich der meisten Qualifikationen sorgte zudem für ein beachtliches Macht- und Drohpotenzial.

Neben der umfangreichen Gruppe von Berufsbildungsakteuren und den staatlichen Akteuren an der Spitze des Arbeitskreises waren zwar einige Vertreter der anderen Bildungsbereiche im AK DQR vertreten, diese waren jedoch in vielerlei Hinsicht benachteiligt und hatten im Entwicklungsprozess offenkundig nur randständige Positionen inne. Hier spielte es sicherlich auch eine gewisse Rolle, dass die Bearbeitung der non-formalen und informellen Bildungsergebnisse auf einen späteren Zeitpunkt verschoben wurde.

In einer komplexen Melange aus engem Zeitplan, inhaltlich überaus voraussetzungsvoller Thematik und heterogener Akteurskonstellation konnten sich schließlich die Vertreter der Berufsbildung weitgehend durchsetzen. Damit wurde die Gestaltungsunfähigkeit des Staates von den nichtstaatlichen Akteuren kompensiert. Der am 22. März 2011 vorgelegte, konsensual verabschiedete Deutsche Qualifikationsrahmen (BMBF 2011) ist in mehreren Dimensionen innovativ. Gerade aus der Perspektive nichtakademischer Bildung ist die im DQR festgeschriebene generelle Gleichwertigkeit von Qualifikationen aus verschiedenen Teilen des Bildungssystems ein erheblicher Fortschritt: Alle Qualifikationsstufen sind grundsätzlich auch ohne akademische Ausbildung erreichbar. Im internationalen Vergleich ist feststellbar, dass dem DQR ein eigenständiger Kompetenzbegriff zugrunde liegt, der insbesondere dem aus Sicht der beruflichen Bildung zentralen Konzept der Handlungskompetenz einen hohen Stellenwert einräumt. Zudem nutzt der DQR ein alternatives Deskriptorenset für Bildungsergebnisse, bei dem ebenfalls Einflüsse aus dem Bereich der beruflichen Bildung offenkundig sind: Anstelle der Trias aus „knowledge, skills and competences“ werden Bildungsergebnisse in Deutschland fortan mit zwei Doppel-Deskriptoren beschrieben. Dem Begriffspaar „Wissen und Fertigkeiten“ (zusammen „Fachkompetenz“) wurde mit „Sozial- und Selbstkompetenz“ (zusammen „Personale Kompetenz“) ein zweites Paar zu Seite gestellt, das den DQR bislang international einzigartig macht. Auch innerhalb des deutschen Bildungssystems wird dies vermutlich folgenschwer sein, insofern die Beurteilungslogik der beruflichen Bildung für andere Bildungsbereiche verbindlich wird. Mit Ausnahme der Hochschule (hier finden wie beschrieben die Dublin-Deskriptoren Anwendung) werden fortan Bildungsergebnisse aus allen Bereichen anhand der erreichbaren Handlungskompetenz beurteilt werden.

\section{Fazit}

Am Beginn dieses Beitrags stand die Frage, wie es um die Reformfähigkeit der deutschen Bildungspolitik bestellt ist. Zwischen dem in vielen Studien belegten und seit einigen Jahren auch in der öffentlichen Diskussion stark betonten Reformbedarf in der Gestaltung des gesamten Bildungswesens in Deutschland und der Beschaffenheit des Politikfeldes besteht ein gewisses Dilemma: Die erhebliche Politikverflechtung und das daraus resultierende hohe Vetopotenzial machen Steuerungsprozesse aufwendig, verkomplizieren die Aushandlung von Lösungen und führen insgesamt zu überaus stabilen, kaum reformfähigen Konstellationen - soweit zumindest die Arbeiten von Scharpf $(1985 ; 2009)$ und Tsebelis (2002).

Anhand zweier Fallstudien zu aktuellen bildungspolitischen Reformprojekten konnten wir zeigen, dass es unter vermeintlich reformfeindlichen Bedingungen dennoch zu innovativen Politikergebnissen kommen kann - auch wenn, wie im Fall der deutschen EQR-Umsetzung, gemeinsame Reformziele und monetäre Anreize zur Einigung weitgehend fehlen. Mehr noch: Die Ergebnisse lassen den Schluss $\mathrm{zu}$, dass der erreichte Innovationsgrad sowohl bei der Exzellenzinitiative als auch beim Deutschen Qualifikationsrahmen für lebenslanges Lernen durch die komplexe Kompetenzverteilung erheblich gesteigert wurde. Trotz unterschiedlicher Ausgangslage weisen beide Fälle eine klare Parallele darin auf, dass das erzielte Ergebnis die ursprüngliche Verhandlungsvorlage signifikant übertrifft. Die Einbindung von Vetospielern in den Politikprozess bedeutete also keine Reduzierung des Reformpotenzials, sondern vielmehr dessen Steigerung.

Betrachtet man abseits der Ergebnisse der beiden Reformen den dahinter liegenden Politikprozess, treten gleichwohl durchaus problematische Momente der föderalen Entscheidungsstrukturen zutage: In beiden Fallbeispielen gefährdete die Interaktion der Vetospieler die Aushandlung der Reformen zumindest temporär. Dass dennoch bei- 
de Reformprozesse erfolgreich und sehr innovativ weitergeführt werden konnten, zeigt eindrucksvoll die Einigungsfähigkeit der Vetospieler: Strategien der Konfliktminimierung wird klar der Vorzug vor den unabsehbar hohen politischen Kosten einer Blockade gegeben.

\section{LITERATUR}

Baethge, M. (2008): Das berufliche Bildungswesen in Deutschland am Beginn des 21. Jahrhunderts, in: Cortina, K. S. (Hrsg.): Das Bildungswesen in der Bun desrepublik Deutschland. Strukturen und Entwicklungen im Überblick, Reinbek, S. 541-597

Bandelow, N. (2005): Kollektives Lernen durch Vetospieler? Konzepte britischer und deutscher Kernexekutiven zur europäischen Verfassungs- und Währungspolitik, Baden-Baden

Benz, A. (2003): Konstruktive Vetospieler im Mehrebenensystem, in: Mayntz, R./Streeck, W. (Hrsg.): Die Reformierbarkeit der Demokratie. Innovationen und Blockaden, Frankfurt a. M., S. 205-236

Benz, A. (2009): Ein gordischer Knoten der Politikwissenschaft? Zur Vereinbarkeit von Föderalismus und Demokratie, in: Politische Vierteljahresschrift 50 (1), S. 3-22

Bundesministerium für Bildung und Forschung (BMBF) (2011): Deutscher Qualifikationsrahmen für lebenslanges Lernen, Online unter http://www.deutscherqualifikationsrahmen.de/de?t=/documentManager/sfdoc.file.

supply\&fileID=1323248009368 (letzter Zugriff: 26.04.2012)

Bundesregierung und die Regierungen der deutschen Bundesländer (2005):

Bund-Länder-Vereinbarung gemäß Artikel 91 b des Grundgesetzes (Forschungsförderung) über die Exzellenzinitiative des Bundes und der Länder zur Förderung von Wissenschaft und Forschung an deutschen Hochschulen. Exzellenzvereinbarung (ExV) vom 18. Juli 2005, http://www.wissenschaftsrat.de/ download/Exzellenziniative_Dokumente/BLK-ExIni.pdf (letzter Zugriff: 26.04.2012)

Busemeyer, M. (2006): Der Kampf um knappe Mittel. Die Bestimmungsfaktoren der öffentlichen, privaten und sektoralen Bildungsausgaben im internationalen Vergleich, in: Politische Vierteljahresschrift 46 (3), S. 393-418

Busemeyer, M. (2009): Wandel trotz Reformstau. Die Politik der beruflichen Bildung seit 1970, Frankfurt a. M./New York

Busemeyer, M./Trampusch, C. (2011): Comparative Political Science and the Study of Education (Review Article), in: British Journal of Political Science 41 (2), S. 413-443

Europäisches Parlament und Rat (2008): Empfehlung des Europäischen Parlamentes und des Rates vom 28. April 2008 zur Einrichtung eines Europäischen Qualifikationsrahmens für lebenslanges Lernen (2008/C111/01), http://eur-lex. europa.eu/LexUriServ/LexUriServ.do?uri=OJ:C:2008:111:0001:0007:DE:PDF (letzter Zugriff: 26.04.2012)

Förster, C. (2011): Nationale Hochschulpolitik im Europäischen Hochschulraum Studiengebühren und Hochschulgovernance in Deutschland, England und Österreich (1999-2009), Dissertation an der Eberhard Karls UniversitätTübingen Griessen, D./Braun, T. (2010): Hochschulföderalismus zwischen Kooperationszwang und Blockadegefahr: Deutschland und die Schweiz im Vergleich, in: Trampusch, C./Busemeyer, M., a .a. O., S. 715-746

Hartmann, M. (2006): Die Exzellenzinitiative. Ein Paradigmenwechsel in der deutschen Hochschulpolitik, in: Leviathan 34 (4), S. 447-465

Hrbek, R. (2001): Die föderale Ordnung. Anspruch und Wirklichkeit, in: Recker, M. L./Jellonnek, B./Rauls, B. (Hrsg.): Bilanz: 50 Jahre Bundesrepublik Deutschland, St. Ingbert, S. 53-68

Klenk, J. (2011): Selbstkoordination statt staatlicher Gestaltung - die Entwicklung des Deutschen Qualifikationsrahmens als Prototyp neuer Bildungspolitik?, in: Schmid, J./Amos, K./Schrader, J./Thiel, A. (Hrsg.): Welten der Bildung? Vergleichende Analysen von Bildungspolitik und Bildungssystemen, BadenBaden, S. 223-244

Kreft, J. (2006): Gewerkschaften und Spitzenverbände der Wirtschaft als bildungspolitische Akteure. Positionen, Strategien und Allianzen, Wiesbaden
Meuser, M./Nagel, U. (2009): Experteninterviews - vielfach erprobt - wenig bedacht. Ein Beitrag zur qualitativen Methodendiskussion, in: Bogner, A./Littig, B./Menz, W. (Hrsg.): Das Experteninterview. Theorie, Methode, Anwendung, Wiesbaden, S. 71-94

Münch, R. (2007): Die akademische Elite. Zur sozialen Konstruktion wissenschaftlicher Exzellenz, Frankfurt a. M.

Münch, U. (2009): Von Leuchttürmen, Bildungsgipfeln und Konjunkturtälern. ZurTopographie des deutschen Bildungsföderalismus, in: Europäisches Zentrum für Föderalismus-Forschung (Hrsg.): Jahrbuch des Föderalismus Bd. 10, Baden-Baden, S. 225-235

Pasternack, P. (2008): Die Exzellenzinitiative als politisches Programm - Fortsetzung der normalen Forschungsförderung oder Paradigmenwechsel?, in: Bloch, R./Keller, A./Lottmann A./Würrmann, C. (Hrsg.): Making Excellence. Grundlagen, Praxis und Konsequenzen der Exzellenzinitiative, Bielefeld, S. $13-36$

Powell, J./Bernhard, N./Graf, R. (2012): The Emergent European Model in Skill Formation: Comparing Higher Education and Vocational Training in the Bologna and Copenhagen Processes, in: Sociology of Education, OnlineFirst, veröffentlicht am 03.01., http://soe.sagepub.com/content/early/2012/01/03/ 0038040711427313.full.pdf+html (letzter Zugriff: 26.04.2012)

Sandberger, G. (2008): Die Umsetzung der Föderalismusreform im Hochschulbereich, in: Europäisches Zentrum für Föderalismus-Forschung (Hrsg.): Jahrbuch des Föderalismus Bd. 9, Baden-Baden, S. 160-171

Scharpf, F. W. (1985): Die Politikverflechtungs-Falle: Europäische Integration und deutscher Föderalismus im Vergleich, in: Politische Vierteljahresschrift 26 (4), S. 323-356

Scharpf, F. W. (2000): Interaktionsformen. Akteurzentrierter Institutionalismus in der Politikforschung, Wiesbaden

Scharpf, F. W. (2009): Föderalismusreform. Kein Ausweg aus der Politikverflechtungsfalle?, Frankfurt a. M.

Scharpf, F. W./Reissert, B./Schnabel, F. (1976): Politikverflechtung. Theorie und Empirie des kooperativen Föderalismus in der Bundesrepublik, Kronberg Sozialdemokratische Partei Deutschlands (SPD) (2004): Weimarer Leitlinien Innovation, Weimar

Trampusch, C./Busemeyer, M. (2010) (Hrsg.): Berufsbildungs- und Hochschulpolitik in Deutschland, Österreich und der Schweiz, Sonderheft 16 (4) der Schweizerischen Zeitschrift für Politikwissenschaft

Tsebelis, G. (2002): Veto-Players, How Political Institutions Work, New York Witte, J. (2006): Change of degrees and degree of change. Comparing adaptions of European Higher Education Systems in the Context of the Bologna Process, Enschede

\section{AUTOREN}

CHRISTIAN FÖRSTER, Dr., ist Lehrbeauftragter am Institut für Politikwissenschaft der Eberhard Karls UniversitätTübingen und Projektleiter Forschungsförderung/Themenscouting bei der Medien- und Filmgesellschaft Baden-Württemberg, Innovationsagentur für IT und Medien (MFG). Arbeitsschwerpunkte: Bildungspolitik, Forschungsförderung, nordische Länder, Theorien und Ansätze der Politikfeldanalyse.

foerster@mfg.de

JOHANNES KLENK, Diplom Pädagoge, ist Doktorand im Promotionskolleg der Hans-Böckler-Stiftung "International-vergleichende Forschung zu Bildung und Bildungspolitik im Wohlfahrtsstaat" an der Universität Tübingen. Arbeitsschwerpunkte: Bildungspolitik, Mikropolitik, pädagogische Organisationsentwicklung.

johannes.klenk@ifp.uni-tuebingen.de 